\title{
The effects of age and intelligence on the dark-interval threshold'
}

\author{
ROBERT H. POLLACK, ${ }^{2}$ RICHARD I. PTASHNE, AND DOROTHY JEAN CARTER
} INSTITUTE FOR JUVENILE RESEARCH

\begin{abstract}
The threshold for the detection of the dark interval between two flashes of light (DIT) was investigated for 240 school children aged 6-17. It was found that the threshold showed a linear decline with chronological age, a finding attributed to physiological aging of the visual receptor system producing diminished persistence of the initial stimulus. This finding was counter to the expectation that the DIT would also indicate the growing potency of a highemorder cognitive process (temporal integration of stimulus traces) which would have caused the DIT to rise at some point along the chronological
\end{abstract} scale.

Pollack (1969) has put forth a two-process theory to explain age changes in visual perception. One of these processes, nondevelopmental in nature. involves a progressive loss in receptor sensitivity due to physiological aging. Within the framework of this theory those phenomena which show nondevelopmental age changes (Type I) are not correlated with intellectual ability, but appear to be related directly to receptor efficiency and such stimulus variables as intensity, duration, contrast, and orientation. The operation of all these variables depends on the sensitivity and efficiency of the visual receptor system. Weale (1963) has shown that lenticular density and pigmentation, as well as retinal pigmentation, increase with age, while pupil size decreases. The pinpointing of the precise mechanisms involved, however, requires further physiological investigation. Some behavioral research exists that tends to confirm the above notions. Pollack's investigation of contour detectability thresholds in childhood (1963c) supports the above. Both sensitivity to contour as produced by brightness contrast and the magnitude of the Mueller-Lyer illusion were found to decline between ages 8 and 12 , and intelligence played no significant role. Two experiments by Pollack (1965a, b) on backward figural masking showed that figure grounu contrast of the target and contour orientation of the target and mask were significant variables in producing the masking effect with adults. Those conditions that produced the maximal amount of backward masking in adults were presented to children ranging in age from 7 to 10 years. The interstimulus interval permitting masking declined steadily as a function of increasing chronological age. Adult Ss exhibited masking with even shorter intervals than the 10year-olds. There was no correlation with IQ. Again, stimulus variables interacting with the declining sensitivity of the visual receptor system were believed to be the factors responsible for the decline in masking with age.

The other process put forth by Pollack (1969) is "developmental" in that age changes seem to be related to central cognitive functions and abilities that are hierarchically organized. Those phenomena showing developmental age changes (Type II) are correlated with intellectual ability and do not depend as much upon classical stimulus variation. Presentation of such phenomena usually involves a sequence of discrete stimulus events, registered by the $S$ as traces, which are then centrally compared and contrasted. Pollack (1964) demonstrated this by converting a Type I illusion (MuellerLyer) into a Type II illusion, by altering the mode of presentation from simultaneous to successive. This was done by presenting the oblique inducing lines before presenting the main line. The classical illusion reversed its usual direction; the new illusion increased in magnitude with age, and showed a positive correlation with intelligence. These results, exactly the opposite of those obtained when the same stimuli are presented simultaneously, were attributed to the apparent functioning of a higher-order cognitive process (integration through time) in the perception of the illusion.

It is assumed that in making judgments about two stimuli presented in rapid succession, the individual early in life makes use of the physical persistence of the initial stimulus to make what is virtually a simultaneous comparison. If this stimulus persistence weakens as a function of aging in the receptor system, comparison may come to be based upon the higher-order cognitive function of comparing the trace of the vanished initial stimulus with the second stimulus. This transition was demonstrated by Pollack (1966) in a study dealing with apparent movement. The temporal range of apparent movement decreased between the ages of 6 and 9 , but increased again beyond the age of 9 . Correlation with IQ was nonsignificant for the 6- to 9-year-olds, but positive and significant for 10 and 11 -year-olds. The pattern of results obtained in this experiment was very similar to that obtained in an earlier study by Pollack (1960) concerning the frequency of occurrence of figural aftereffects following brief inspection.

The present experiment was designed to test the hypothesis that stimulus persistence declines with age. The dark-interval threshold (DIT) can be thought of as a simplified version of the critical flicker frequency (CFF), in which only two flashes of light are presented in quick temporal succession with a varying interflash interval. The major physical difference between the two is that in CFF stimuli are presented repeatedly in long trains of flashes. The $S$ is simply asked to report whether he can detect a dark interval between the flashes. At the present time data on the DIT are very scarce. In general, results from studies of CFF (Brown, 1965) have shown that CFF decreases with age. The decrease has been attributed to a decrease in the flexibility of the ciliary muscles controlling pupillary size, increased opacity of the lens, yellowing of the lens, and decrease in the responsiveness of the nervous system, both with respect to regulation of pupil size and in general. Because of the age grouping of earlier CFF studies it is possibly only to say that the decline starts at about age 13. The implication of these earlier studies is that the reduced efficiency of the visual receptor system and the CNS are responsible for the decrease in CFF with age.

Cross (1963) performed a study of CFF with an age range of 6 to 12 years. She found a significant increase in CFF (better performance) with age. Correlation between CFF and IQ was not significant in her 6-8- and 10-year-old groups, but it was significant with her 12-year-old group. If stimulus persistence does weaken as a function of physiological aging of the receptor system, one would expect this increase in performance since it would allow for better resolution of flashes. This study also indicates that by puberty intelligence may begin to play a role, and that CFF begins to decline beyond the age of 12 , possibly as the cognitive function of taking into account traces of initial stimuli interferes with the perception of subsequent stimuli. This above notion is still 
rather tentative due to the unknown relationship of CFF to intelligence. Positive, negative, and zero correlations have been obtained between $\mathrm{CFF}$ and a variety of intelligence tasks (Landis \& Hamwi, 1956; Wilson, 1963).

In formulating expectations about the DIT we are faced with alternatives because of the lack of, and the inconclusiveness, of the evidence. The inability to anticipate the sensitivity with which the measure of the DIT will indicate the declining physical sensitivity of the visual receptor system, or pick up the onset of a higher-order cognitive process (temporal integration of stimulus traces), makes prediction difficult.

If the sensitivity of the measure of the DIT is such that it will reflect the loss of efficiency of the visual receptor system, and no more, then we would expect that as stimulus persistence does decrease with age, the DIT would also decrease with age, as the persistence of the initial stimulus bridges less and less of the dark interstimulus interval.

If the DIT should be sensitive enough to reflect the onset of a higher-order cognitive process, then the expectation would be different. We would once again expect the DIT to decrease with age, due to a decrease in the persistence of the initial stimulus. At some point in the age range, however, as stimulus persistence continues to decline, a new analogous process, the interfering presence of the trace of the initial stimulus, could replace it, perhaps causing the DIT to rise as age increases further.

\section{SUBJECTS 3}

Two-hundred-forty suburban school children, aged 6-17 years, served as Ss. Each age group was represented by 10 males and 10 females, all with 20/30 uncorrected vision or better, as determined by the Master Orthorater.

\section{APPARATUS}

Flashes were presented binocularly in a three-channel electronic tachistoscope (Model G) manufactured by Scientific Prototype Manufacturing Corporation of New York. Channel 1 and a back lighter matched for brightness to Channel 1 served as the flash sources. Each flash was produced by passing illumination through an aperture $6 \mathrm{~mm}$ in diam cut from the center of a black background card. The visual angle of the flash was $18 \mathrm{~min} 40 \mathrm{sec}$. Flash 1 was generated from Channel 1 , followed by a period of no illumination (dependent variable). Flash 2 was generated by the back lighter. Both stimuli were thus presented through the same aperture. The flash intensity level used was $8.84 \mathrm{ft}-\mathrm{c}$, as measured by a Macbeth Illuminometer at the eyepiece. A preliminary experiment (Pollack, Carter, \& Ptashne, 1968) had shown no differences due to brightness. It was decided, therefore, to use the maximum brightness permitted by the tachistoscope.

\section{PROCEDURE}

Each $\mathrm{S}$ was dark-adapted for $5 \mathrm{~min}$. He was then shown examples of obviously double and single flashes. He was instructed to report " 2 " whenever he could detect a dark interval between the flashes, and to report " 1 " when he saw one flash or failed to detect any dark interval. The light source was turned on periodically between trials to provide a target for $S$ in the middle of the visual field. Thresholds were obtained from five ascending and five descending series presented in counterbalanced order, with interflash intervals ranging from 0 to $250 \mathrm{msec}$ presented in 10 -msec steps. A series ended when $S$ reported three consecutive " $2 s$ " or " $1 \mathrm{~s}$." The three brightness conditions were given to all $\mathrm{Ss}$ in counterbalanced order. Flash Durations 1 and 2 were kept constant at 20 msec.

\section{RESULTS}

Since an initial analysis revealed no sex differences, boys' and girls' scores were combined for all subsequent analyses. Each $S$ had one score, the average threshold obtained in one session. Analysis of variance showed a significant age effect ( $F=4.47$, df $=11 / 228, p<.001)$. However, inspection of Fig. 1 shows that there was an upward turn in the curve between the ages of 135 months and 158 months. Since the determination of the significance or nonsignificance of this upturn is important in the theoretical explanation of the outcome of this experiment, a trend analysis (Winer, p. 70ff) was performed. This showed a significant linear component $(F=32.46$, df $=1 / 228, p<.001)$, with no significant deviations from linearity $(F=1.68$, $\mathrm{df}=10 / 228, \mathrm{p}<.001)$. The line of best fit for the data is described by the equation $Y^{\prime}=-.2134 X+107.83$. Hence the DIT can best be described as diminishing in a linear fashion with age.

To complete the analysis of the relative contributions of age and intelligence, ${ }^{4}$ correlations were calculated. Correlation of DIT with CA was $-.23(p<.01)$, while that with MA was nonsignificant $(r=-.05)$. CA was highly correlated with MA $(r=.896, p<.001)$. Thus it is seen that only age is significantly related to the decline in DIT.

\section{DISCUSSION}

It seems that the explanation based on the decline in stimulus persistence with age can best support the results obtained in this experiment. The fact that there was a clear linear decline in DIT with age supports this argument. Perhaps, physiologically, the visual receptor system is at its peak efficiency in the younger years and, due to the various physiological changes mentioned earlier, it gradually loses some of its sensitivity. Assuming that central registration of visual stimulus input is directly related to the sensitivity of the visual receptor system, it could be expected that stimulus persistence would be a phenomenon declining with age. It appears that the DIT has proven to be a successful behavioral measure for plotting the course of stimulus persistence. In our specific case, in younger children the input of the first flash might have persisted longer into the dark interval, hence causing them to report one flash of light at greater interflash intervals than did the older children. As the stimulus persistence of the first flash of light bridges less and less of the dark interval between the two flashes, it becomes easier for the $S$ to detect this dark interval, and therefore to report two flashes. It should be emphasized again that more work needs to be done by physiologists to identify the actual changes within the visual receptor system and their ontogenetic course. Once this body of knowledge is obtained, further behavioral work can be done to specify deficits.

It also was expected that as stimulus persistence declined with age, comparison of stimuli would begin to be based upon the higher-order cognitive function of comparing the trace of the vanished initial stimulus with the second stimulus. It has already been shown that this did not happen in our experiment. The almost zero correlation between DIT and MA further supports the notion that the DIT measure has not tapped any intellectual functioning. It cannot be denied that the process of "temporal integration" exists; the answer as to why it has not been demonstrated here must lie in the stimulus situation.

Perhaps the mechanism responsible for temporal integration in the higher sensorium has some threshold value that has to be reached in order to trigger it. This threshold may depend upon certain stimulus variables that have not been satisfied by the present paradigm. In Pollack's $(1960,1966)$ two studies of apparent movement and figural aftereffects, where the 
transition from perception based on stimulus persistence to that based on temporal integration of memory traces was demonstrated, there were some stimulus similarities with the present study, but also two outstanding differences, that could account for the failure to call temporal integration into play. In both earlier studies the initial stimulus duration was longer than in the present study. Perhaps this value in our present study $(20 \mathrm{msec})$ was below a threshold for establishing trace. In addition, in the apparent movement study a natural by-product of the phenomenon is a lateral displacement of the stimulus across the visual field, produced by stimulating contiguous areas of the visual cortex. It might be that some spatial displacement is necessary for the demonstration of "temporal integration."

It is more difficult to account for the disagreement with CFF studies of age changes beyond 13. The only suggestion that is offered is that the continuous repetition of stimulation involved in CFF experiments might possibly trigger the trace-registration/trace-integration mechanism. By considering some of these factors, it is possible that the present stimulus paradigm could be modified to demonstrate the phenomenon of temporal integration of memory traces. In any case, the present study has shown that stimulus persistence as measured by the DIT is a declining function of age.

\section{REFERENCES}

BROWN, J. L. Flicket and intermittent stimulation. In C. H. Graham et al (Eds.), Vision and Visual Perception. New York: John Wiley \& Sons, Inc., 1965. Pp. 251-320.

CROSS, J. P. Relation of age and mental growth to the CFF response in children. Child Development, 1963, 34, 739-744.

LANDIS, C., \& HAMWI, V. Critical flicker frequency, age, and intelligence. Gerontologia, 1963, 7, 200-208.
POLLACK, R. H. Figural aftereffects as a function of age. Acta Psychologica, 1960, 17, 417-423.

POLLACK, R. H. Contour detectability thresholds as a function of chronological age. Perceptual \& Motor Skills, 1963, 17, 41 1-417.

POLLACK, R. H. Simultaneous and successive presentation of elements of the Mueller-Lyer figure and chronological age. Perceptual \& Motor Skills, 1964, 19, 303-310.

POLLACK, R. H. Effects of figure-ground contrast and contour orientation on figural masking. Psychonomic Science, 1965a, 2, 369-370.

POLLACK, R. H. Backward figural masking as a function of chronological age and intelligence. Psychonomic Science, 1965b, 3 , $65+66$.

POLLACK, R. H. Temporal range of apparent movement as a function of age and intelligence. Psychonomic Science, 1966, 5, 243-244.

POLLACK, R. H. Some implications of ontogenetic changes in perception. In D. Elkind and J. Flavell (Eds.), Essays in cognitive development: Studies in honor of Jean Piaget. New York: Oxford University Press, 1969.

POLLACK, R. H., PTASHNE, R. I., \& CARTER, D. J. The dark-interval throshold as a function of age. Psychonomic Science, 1968, 12, 237-238.

WEALE, R. A. The aging eye. Great Britain: Adlark \& Son Ltd., Bartholomew Press (Harper \& Row), 1963.

WINER, B. J. Statistical principles in experimental design. New York: McGraw-Hill, 1962.

\section{NOTES}

1. This research was supported by Grant No. HD 01433 awarded by the National Institute of Child Health and Human Development.

2. Address: 232 East Ohio St., Chicago, Illinois 60611.

3. We are grateful for the cooperation of Mr. Darrell Holsteen, Superintendent of School District 89, Glen Ellyn, illinois, Mr. Ronald Howard, Principal of Arbor View School, and Mr. John Sheahan, Principal of Glenbard West High School, Glen Ellyn, Illinois.

4. The measure of intelligence was the Lorge-Thomdike IQ Test. Scores were not available for $S s$ in the 6- and 7-year-old groups. (Accepted for publication December 30, 1968.) 